EPJ Web of Conferences 73, 03007 (2014)

DOI: $10.1051 /$ epjconf/20147303007

(C) Owned by the authors, published by EDP Sciences, 2014

\title{
b and c spectroscopy at LHCb
}

\author{
Patrizia de Simone ${ }^{\mathrm{a}}$ on behalf of the LHCb Collaboration \\ Laboratori Nazionali di Frascati, I.N.F.N., via E. Fermi 40, 00044 Frascati (Rome), Italy
}

\begin{abstract}
The $\mathrm{LHCb}$ experiment is designed to study the decays and properties of heavy flavoured hadrons produced in the forward region from $p p$ collisions at the CERN Large Hadron Collider. It has recorded the world's largest data sample of beauty and charm hadrons, enabling precise studies into the spectroscopy of such particles, including discoveries of new states and measurements of their properties such as masses, width and quantum numbers. The latest results in this area are reviewed.
\end{abstract}

\section{Introduction}

The LHCb detector [1] is optimized for measurements in heavy-flavour physics at the Large Hadron Collider LHC, at CERN. As the $b \bar{b}$ pairs are predominantly produced in the same forward or backward direction at high energies, the LHCb detector is a single forward arm spectrometer with an unique pseudo-rapidity acceptance of $2<\eta<5$. The LHCb experiment has accumulated $\simeq 1 \mathrm{fb}^{-1}$ of data at $\sqrt{s}=7 \mathrm{TeV}$ during the years 2010-2011, and $\simeq 2 \mathrm{fb}^{-1}$ at $\sqrt{s}=8 \mathrm{TeV}$ during the 2012 .

\section{2. c-hadrons}

\section{$2.1 X(3872)$ quantum numbers}

The $X(3872)$ particle (called $X$ in this section) was discovered in 2003 by the Belle experiment [2] and subsequently observed by several other experiments. Despite a large experimental effort, the nature of this new state is still uncertain. Among the open possibilities are conventional charmonium states and loosely bound $D^{* 0} \bar{D}^{0}$ molecules [3], tetra-quark states [4], or their mixture [5]. Measurements of the quantum numbers are important to shed light on the nature of the $X$.

After measuring the mass and the production cross section [6] LHCb has recently measured the $X$ quantum numbers $J^{P C}$ [7], resolving the observed ambiguity between $1^{++}$and $2^{-+}$[8], in favour of the former. With $\simeq 1 \mathrm{fb}^{-1}$ collected at $\sqrt{s}=7 \mathrm{TeV}$ we have performed the first analysis of the complete five-dimensional angular correlations of $313 \pm 26 B^{+} \rightarrow X K^{+}$decays, where $X \rightarrow J / \psi \pi^{ \pm} \pi^{\mp}$ and $J / \psi \rightarrow \mu^{ \pm} \mu^{\mp}$. The selection is optimized on the more abundant $B^{ \pm} \rightarrow \psi(2 S) K^{ \pm}$similar channel, and the signal yield is given by a fit to the data using a Crystal Ball function for the signal and a linear function for the background, as shown in Fig. 1 left. To discriminate between the $1^{++}$and $2^{-+}$ assignments, we performed a likelihood ratio test that unambiguously reject the hypotheses $J^{P C}=2^{-+}$ at $8.4 \sigma$ (see Fig. 1 right).

\footnotetext{
ae-mail: patrizia.desimone@lnf .infn.it
}

This is an Open Access article distributed under the terms of the Creative Commons Attribution License 4.0, which permits unrestricted use, distribution, and reproduction in any medium, provided the original work is properly cited. 

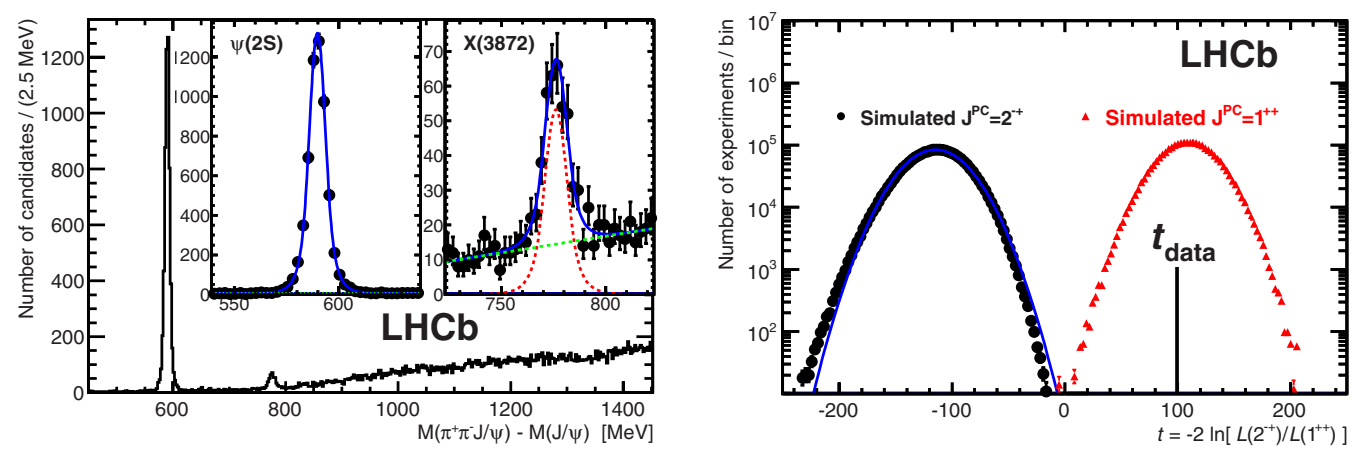

Figure 1. Left: distribution of $m\left(J / \psi \pi^{ \pm} \pi^{\mp}\right)-m(J / \psi)$ for $B^{ \pm} \rightarrow J / \psi \pi^{ \pm} \pi^{\mp} K^{ \pm}$candidates. The results of the fit around the $\psi(2 S)$ and the $\mathrm{X}$ masses are shown in the inserts. The solid blue, dashed red, and dotted green lines are the total, signal and background components, respectively. Right: distribution of the test statistic for the simulated experiments experiments with $J^{P C}=2^{-+}$(black circles) and with $J^{P C}=1^{++}$(red triangles). A Gaussian fit to the $2^{-+}$distribution is overlaid (blue solid line). The value of the test statistic for the data, $t_{\text {data }}$, is shown by the solid vertical line.

\subsection{Precision measurement of the $D^{0}$ mass}

With a data sample of $\simeq 1 \mathrm{fb}^{-1}$ collected at $\sqrt{s}=7 \mathrm{TeV}$, LHCb has measured the mass of the $D^{0}$ meson [9] using the decay $D^{0} \rightarrow K^{+} K^{-} K^{-} \pi^{+}$, where the $D$ mesons are produced in semileptonic $b$-decays: $M\left(D^{0}\right)=1864.75 \pm 0.15$ (stat) \pm 0.11 (syst) $\mathrm{MeV} / \mathrm{c}^{2}$, with the dominant systematic uncertainty coming from the knowledge of the momentum scale. Using the decay modes $D^{0} \rightarrow K^{+} K^{-} \pi^{+} \pi^{-}$and $D_{s}^{+} \rightarrow K^{+} K^{-} \pi^{+}$we measured also the mass differences:

$M\left(D^{+}\right)-M\left(D^{0}\right)=4.76 \pm 0.12($ stat $) \pm 0.07($ syst $) \mathrm{MeV} / \mathrm{c}^{2}$

$M\left(D_{s}^{+}\right)-M\left(D^{+}\right)=98.68 \pm 0.03($ stat $) \pm 0.04($ syst $) \mathrm{MeV} / \mathrm{c}^{2}$.

\subsection{Studies of $D_{j}$ mesons}

The search of the $D_{j}$ mesons has been performed in a data sample of $\simeq 1 \mathrm{fb}^{-1}$ collected at $\sqrt{s}=7 \mathrm{TeV}$, using the inclusive reactions:

$$
p p \rightarrow D^{+} \pi^{-} X, \quad p p \rightarrow D^{0} \pi^{+} X, p p \rightarrow D^{*+} \pi^{-} X
$$

where $X$ represents a system composed of any collection of charged and neutral particles [10].

Due to the availability of the helicity angle information, the fit to the $D^{*+} \pi^{-}$mass spectrum allows a spin analysis of the produced resonances and a separation of the different spin-parity components: we are able to separate our sample in an enhanced unnatural parity sample and a natural parity sample. The $D^{+} \pi^{-}$and $D^{0} \pi^{+}$mass spectra consist of only natural parity resonances, however these final states are affected by cross-feed from all the resonances that decay to the $D^{*} \pi$ final state.

We observed the already well established resonances $D_{1}(2420)^{0}$ in the $D^{*+} \pi^{-}$final state, and $D_{2}^{*}(2460)$ in the $D^{*+} \pi^{-}, D^{+} \pi^{-}$and $D^{0} \pi^{+}$final states, confirming their spin-parity assignments. We also observed two natural parity resonances $D_{J}^{*}(2650)^{0}$ and $D_{J}^{*}(2760)^{+}$in the $D^{*+} \pi^{-}$mass spectrum. The study of the $D^{+} \pi^{-}$and $D^{0} \pi^{+}$mass spectra supports the presence of the $D_{J}^{*}(2760)^{+}$, while the analysis of the $D_{J}^{*}(2650)^{0}$ region is inconclusive because of the cross-feed. Finally the analysis of the $D^{*+} \pi^{-}$final state showes also the presence of two unnatural parity states $D_{J}(2580)^{0}$ and $D_{J}(2740)^{0}$. The measured masses and widths of the observed resonances are listed in Table 1. 
Table 1. Resonance parameters.

\begin{tabular}{llll}
\hline Resonance & Final state & Mass $(\mathrm{MeV})$ & Width $(\mathrm{MeV})$ \\
\hline$D_{1}(2420)^{0}$ & $D^{*+} \pi^{-}$ & $2419.6 \pm 0.1 \pm 0.7$ & $35.2 \pm 0.4 \pm 0.9$ \\
$D_{2}^{*}(2460)^{0}$ & $D^{*+} \pi^{-}$ & $2460.4 \pm 0.4 \pm 1.2$ & $43.2 \pm 1.2 \pm 3.0$ \\
$D_{2}^{*}(2460)^{0}$ & $D^{+} \pi^{-}$ & $2460.4 \pm 0.1 \pm 0.1$ & $45.6 \pm 0.4 \pm 1.1$ \\
$D_{2}^{*}(2460)^{+}$ & $D^{0} \pi^{+}$ & $2463.1 \pm 0.2 \pm 0.6$ & $48.6 \pm 1.3 \pm 1.9$ \\
\hline$D_{J}^{*}(2650)^{0}$ & $D^{*+} \pi^{-}$ & $2649.2 \pm 3.5 \pm 3.5$ & $140.2 \pm 17.1 \pm 18.6$ \\
$D_{J}^{*}(2760)^{0}$ & $D^{*+} \pi^{-}$ & $2761.1 \pm 5.1 \pm 6.5$ & $74.4 \pm 3.4 \pm 37.0$ \\
$D_{J}^{*}(2760)^{0}$ & $D^{+} \pi^{-}$ & $2760.1 \pm 1.1 \pm 3.7$ & $74.4 \pm 3.4 \pm 19.1$ \\
$D_{J}^{*}(2760)^{+}$ & $D^{0} \pi^{+}$ & $2771.7 \pm 1.7 \pm 3.8$ & $66.7 \pm 6.6 \pm 10.5$ \\
\hline$D_{J}(2580)^{0}$ & $D^{*+} \pi^{-}$ & $2579.5 \pm 3.4 \pm 5.5$ & $177.5 \pm 17.8 \pm 46.0$ \\
$D_{J}(2740)^{0}$ & $D^{*+} \pi^{-}$ & $2737.0 \pm 3.5 \pm 11.2$ & $73.2 \pm 13.4 \pm 25.0$ \\
\hline
\end{tabular}

\section{3. b-hadrons}

\subsection{B baryons masses}

At $\mathrm{LHCb}$ we have reconstructed three of the $16 b$-baryons predicted ground states, namely the $\Lambda_{b}^{0}, \Xi_{b}^{-}$ and the $\Omega_{b}^{-}$in their decays $J / \psi \Lambda^{0}, J / \psi \Xi^{-}$and $J / \psi \Omega^{-}$respectively. With a data sample of $\simeq 1 \mathrm{fb}^{-1}$ collected at $\sqrt{s}=7 \mathrm{TeV}$, we have measured the masses [11] of the above mentioned baryons: $M\left(\Lambda_{b}^{0}\right)=$ $5619.53 \pm 0.13$ (stat) \pm 0.45 (syst) $\quad \mathrm{MeV} / \mathrm{c}^{2}, \quad M\left(\Xi_{b}^{-}\right)=5795.8 \pm 0.9($ stat $) \pm 0.4($ syst $) \quad \mathrm{MeV} / \mathrm{c}^{2}$, $M\left(\Omega_{b}^{-}\right)=6046.0 \pm 2.2($ stat $) \pm 0.4($ syst $) \mathrm{MeV} / \mathrm{c}^{2}$. Where the dominant systematic uncertainty comes from the knowledge of the momentum scale. Our $M\left(\Omega_{b}^{-}\right)$result solves the inconsistency between the $\mathrm{CDF}$ and D0 measurements [12] in favour of the former.

\subsection{Excited $\Lambda_{b}^{0}$ baryons}

The quark model predicts the existence of two orbitally excited states $\Lambda_{b}^{* 0}$ with quantum numbers $J^{P}=$ $1 / 2^{-}, 3 / 2^{-}$, that are expected to decay to $\Lambda_{b}^{0} \pi^{+} \pi^{-}$or to $\Lambda_{b}^{0} \gamma$ depending on the mass of the excited states. We search for the excited $\Lambda_{b}^{* 0}$ barions starting from the recontruction of $\Lambda_{b}^{0}$ barions through the decay chain $\Lambda_{b}^{0} \rightarrow \Lambda_{c}^{+} \pi^{-}$and $\Lambda_{c}^{+} \rightarrow p K^{-} \pi^{+}$. The selected sample from $1 \mathrm{fb}^{-1}$ is large (70540土330) and clean $(\mathrm{S} / \mathrm{B} \simeq 11)$, and the background composition is well understood [13]. Combining the $\Lambda_{b}^{0}$ baryons with two oppositely charged pions coming from the same vertex, we reconstruct the total invariant mass to look for the existence of the predicted $\Lambda_{b}^{* 0}$ baryons [14]. We have observed two narrow peaks slight above the $\Lambda_{b}^{0} \pi^{+} \pi^{-}$threshold, that we have interpreted as the $1 / 2^{-}$and $3 / 2^{-}$orbital excitations of the ground state $\Lambda_{b}^{0}$. The masses that we measure, are

$M\left(\Lambda_{b}^{* 0}\left(1 / 2^{-}\right)\right)=5911.97 \pm 0.12 \pm 0.02 \pm 0.66 \mathrm{MeV} / \mathrm{c}^{2}$, $M\left(\Lambda_{b}^{* 0}\left(3 / 2^{-}\right)\right)=5919.77 \pm 0.08 \pm 0.02 \pm 0.66 \mathrm{MeV} / \mathrm{c}^{2}$,

the third error is due to the uncertainty on the $\Lambda_{b}^{0}$ mass. Because of the remarkably good mass resolution, order of $0.2 \mathrm{Mev} / \mathrm{c}^{2}$, we were able to set upper limits on the natural widths $\Gamma\left(\Lambda_{b}^{* 0}\left(1 / 2^{-}\right)\right)<0.66 \mathrm{MeV}$ $(<0.83 \mathrm{MeV})$ and $\Gamma\left(\Lambda_{b}^{* 0}\left(3 / 2^{-}\right)\right)<0.63 \mathrm{MeV}(<0.66 \mathrm{MeV})$ at the $90 \%(95 \%)$ C.L.

\section{$3.3 \Lambda_{b}^{0}$ lifetime}

The Heavy Quark Expansion theory supports the hypothesis that the $b$-hadron lifetimes are quite similar; in the case of the ratio $\frac{\tau\left(\Lambda_{b}^{0}\right)}{\tau\left(\bar{B}^{0}\right)}$ differences of only a few percent are expected [15]. On the experimental side an average of all the available data before 2004 gave $0.786 \pm 0.034$ [16], while recent measurements at Tevatron and LHC [17] could resolve this discrepancy but the uncertanties are still large. LHCb has recently published the measuremet of the $\frac{\tau\left(\Lambda_{b}^{0}\right)}{\tau\left(\bar{B}^{0}\right)}[18]$ with $\simeq 1 \mathrm{fb}^{-1}$ collected at $\sqrt{s}=7 \mathrm{TeV}$. The $\Lambda_{b}^{0}$ barions are selected in the $J \psi p K^{-}$decay channel (note that this is the first 
observation of this decay), while the $\bar{B}^{0}$ mesons are selected using the well known $\bar{B}^{0} \rightarrow J / \psi \pi^{+} K^{-}$ channel. These decay modes have the same topology into 4 charged tracks, facilitating the cancellation of uncertainties. The fit to the ratio of the decay time distributions of the two decay channels, gives us the reciprocal lifetimes difference: $\frac{1}{\tau\left(\Lambda_{b}^{0}\right)}-\frac{1}{\tau\left(\bar{B}^{0}\right)}=16.4 \pm 8.2 \pm 4.4 \mathrm{~ns}^{-1}$ and the ratio of lifetimes is: $\frac{\tau\left(\Lambda_{b}^{0}\right)}{\tau\left(\bar{B}^{0}\right)}=0.976 \pm 0.012 \pm 0.006$ where we use the world average value for $\tau\left(\bar{B}^{0}\right)$ [19]. And finally we determine: $\tau\left(\Lambda_{b}^{0}\right)=1.482 \pm 0.018 \pm 0.012 \mathrm{ps}$, that is consistent with the current world average of $1.429 \pm 0.024$ ps [19].

\section{Conclusion}

In addition to the measurements reported here, there are other recent results from LHCb. Among these, we would like to mention the the observation of a resonance in $B^{+} \rightarrow K^{+} \mu^{+} \mu^{-}$decays at low recoil [20], the observation of the decay $B_{c}^{+} \rightarrow B_{s}^{0} \pi^{+}$[21], and the measurement of the $B_{c}$ lifetime [22]. The $\mathrm{LHCb}$ experiment, with its world class heavy flavour spectroscopy program, will continue to provide precise and competitive measurements.

\section{References}

[1] The LHCb Collaboration, JINST 3, S08005 (2008)

[2] The Belle Collaboration, PRL 93, 072001 (2004)

[3] E. Swanson, PR 429, 243 (2006)

[4] L. Maiani, F. Piccinini, A.D. Polosa and V. Riquer, PRD 71, 014028 (2005)

[5] C. Hanhart, Y. Kalashnikova, and A. Nefediev, EPJ A 47, 101 (2011)

[6] The LHCb Collaboration, EPJ 72, 1972 (2012)

[7] The LHCb Collaboration, PRL 110, 222001 (2013)

[8] The BaBar Collaboration, PRD 82, 011101 (2010); The Belle Collaboration, PRD 84, 052004 (2011)

[9] The LHCb Collaboration, JHEP 1306, 065 (2013)

[10] The LHCb Collaboration, JHEP 09, 145 (2013)

[11] The LHCb Collaboration, PRL 110, 182002 (2013)

[12] The CDF Collaboration, PRL 101, 232002 (2008), The D0 Collaboration, PRD 80, 072003 (2009)

[13] The LHCb Collaboration, LHCB-CONF-2011-036

[14] The LHCb Collaboration, PRL 109, 172003 (2012)

[15] H.Y. Cheng, PRD 56, 2783 (1997); M. Neubert and C.T. Sachrajda NP, B 483, 339 (1997); J.L. Rosner, PLB 379, 267 (1997)

[16] C. Tarantino, EPJC 33, S895 (2004)

[17] The ATLAS Collaboration, PRD 87, 032002 (2013); The CDF Collaboration, PRL 106, 121804 (2011); The D0 Collaboration, PRD 85, 112003 (2012)

[18] The LHCb Collaboration, PRL 111, 102003 (2013)

[19] Particle Data Group, PRD 86, 010001 (2013)

[20] The LHCb Collaboration, PRL 111, 112003 (2013)

[21] The LHCb Collaboration, PRL 111, 181801 (2013)

[22] The LHCb Collaboration, arXiv:1401.6932, submitted to EPJC 